\title{
Reciprocal Chromosome Translocations: Analysis of Two Mutants by Interactive Computer*
}

\author{
RUSSELL W. DICKERSON, NGUYEN-HUU XUONG, and OLIVER W. JONES
}

Departments of Medicine and Physics, University of California, San Diego, USA

During recent years, efforts in several laboratories have been directed towards automated analysis by computer of chromosome patterns (Butler, Butler, and Stroud, 1968; Cochrane, 1968; Wald and Preston, 1968; Frey, 1969; Hilditch and Rutovitz, 1969; Mendelsohn et al, 1969; Neurath and Brand, 1969; Ruddle et al, 1969; Gallus and Neurath, 1970; Castleman, 1972). Although it has been possible to achieve chromosome measurements by computer techniques, automatic karyotyping, ie, pairing chromosomes within a major group, has not been consistent. Other difficulties have made automated chromosome analysis still impractical for routine use. For example, in a fast, fully automated program which takes about $3 \mathrm{~min} /$ cell, the rate of error in locating the centromere is too high (about $10 \%$ ) for karyotyping (Gallus, Montanaro, and Maccacaro, 1968). On the other hand, the method described here, which allows interaction between the computer and an operator in a manner similar to Neurath and Brand (1969) will reduce this error but requires considerable time $(20 \mathrm{~min} /$ cell). Thus it seems reasonable to state that at present automated procedures for chromosome analysis are not yet applicable to large routine measurements. Nevertheless, computer analysis can be utilized to complement the visual interpretation of special chromosome abnormalities. For example, in prenatal diagnosis of chromosome aberrations, visual analysis may raise questions answerable by computer measurements. In the specific instance of balanced reciprocal translocation carriers, all adjunctive information is useful in determining whether a translocation has become unbalanced in the progeny.

The translocation mutants are of special interest in human cytogenetics. Since balanced translocation, either centric fusion or reciprocal, invariably results in no detectable phenotypic abnormality in the indi-

\footnotetext{
Received 31 May 1972.

* Reprint requests to: Oliver W. Jones, MD, Department of Medicine, Basic Science Building, University of California, San Diego, La Jolla, California 92037, USA.
}

vidual, the true frequency of chromosome translocations in the human population is not known. Recently the work of Lubs and Ruddle (1970) suggested that the frequency of translocation mutations per 1000 population in a large series of human subjects is approximately $1 \%$.

From a genetic standpoint, one of the important sequelae of a balanced translocation in one of the parents is the increased risk of malsegregation with resulting abnormalities in the progeny (Lejeune, Dutrillaux, and de Grouchy, 1970; Macintyre, 1971). On the other hand, it is known that reciprocal translocations can be transmitted for generations with no phenotypic evidence for malsegregation. With the increasing application of prenatal diagnosis by analysis of cultured amniotic cells, decisions regarding malsegregation of translocations will have to be made without benefit of seeing the fetal phenotype. Thus the use of all adjunctive information to make a secure diagnosis in cases at risk for chromosome translocation will be important. It is the purpose of this paper to describe 2 new translocation defects we have encountered and to show that computer measurements of chromosome patterns may be a useful tool in the analysis of chromatin exchange in translocations.

\section{Materials and Methods}

Cell Cultures. All cultures were performed on cells cultured from peripheral blood following well established procedures (Hungerford, 1965).

Chromosome Analysis. The chromosome spread is photographed through a Zeiss brightfield microscope and the resulting micrograph digitized on a rotating drum film scanner which is connected to an IBM 1800 computer. This system has been described elsewhere (Xuong, 1969). Other hardware components used are a Tektronix 611 storage scope for chromosome boundary display and an interactive device which allows the operator to communicate with the computer. When all chromosomes have been processed, the results are presented on a graph using a CalComp plotter. 
The computer programs for chromosome measurement can be divided into 4 parts.

(1) The SPECT program uses a method employed by Mendelsohn, Mayall, and Prewitt (1968) to determine the threshold grey level which, as much as possible, will divide the metaphase into discrete intact chromosomes.

(2) The rough search program (FIND) locates the approximate boundaries of all bodies in the picture with grey levels lower than the threshold value (Ledley, 1964). FIND makes a plot similar to Fig. 1 where the numbers represent the order in which the body was located by the program and are used later for chromosome identification.

(3) Program FINE makes measurements on each chromosome. For each body found by FIND, FINE

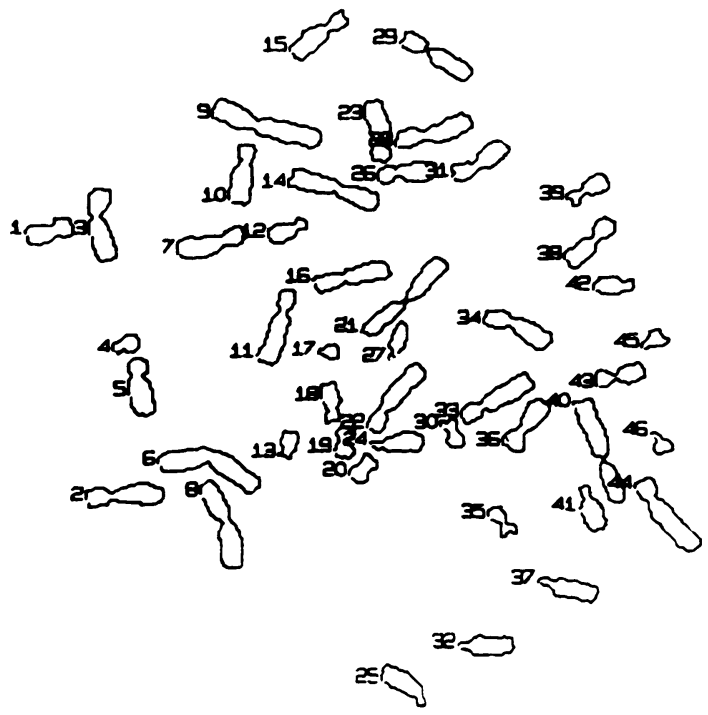

Fig. 1. Computer reproduction and numerical identification of metaphase chromosomes.

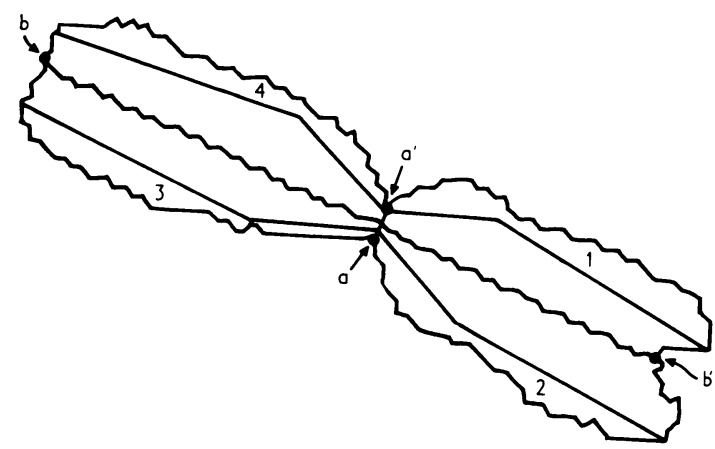

Fig. 2. Computer reproduction of a single metaphase chromosome. The solid line represents the threshold grey level locus and each arm is numbered. $\quad a-a^{\prime}=$ the line segment across the minimum constriction in the centromere region. $b-b^{\prime}$ along with $a-a^{\prime}$ divides the chromosome into four regions that can be identified with the arms. locates the exact boundary and plots it on the storage scope (Fig. 2). The operator will either recognize the body as a chromosome or reject it as a piece of dust or an interphase cell. Once a positive decision is made, the most important function of the operator is to recognize the centromere position and transmit it to the computer through the interactive device. If the chromosome is metacentric, the operator enters two points approximately on either side of the minimum constriction. The computer will automatically find the minimum constriction (points a and $a^{\prime}$ in Fig. 2) and place the centromere in the middle. In the case of the acrocentric chromosomes like the $Y$ or those in groups $D$ and $G$, we arbitrarily place the centromere on the boundary near the one point entered by the operator.

From this point on, the measurements are completely automatic. Since the chromosome is symmetrical, the computer knows the rough position of the boundary point between the arm pairs. The exact position of this point is found by searching for the minimum distance between the centromere and a few boundary points in this region (points $b$ and $b^{\prime}$ in Fig. 2). The line segments joining the centromere to the points $a, a^{\prime}, b$, and $b^{\prime}$ divide the chromosome into 4 regions that can be identified with the 4 arms. To find the arm length, the program first locates the end of each arm by picking the farthest point in the arm from the centromere. A hinge point is needed, particularly in the presence of binding of a chromosome arm, to provide good measurements. We arbitrarily locate this point at about one third of the arm from the centromere. The arm length is defined as the sum of the distances between the centromere region and the hinge point, and between the hinge point and the end of the arm.

(4) After all the bodies have been processed (Fig. 3a), a computer program summarizes the results in a plot (Fig. 3b), where each chromosome is represented by a circle whose position is determined by the total length on the abscissa and the ratio calculated from the length of the short arms divided by total length on the ordinate. To make the plot axes universal, we normalize the total length of each chromosome with the summation of total lengths for the whole cell including the sex chromosomes (Mendelsohn et al, 1966).

Although our method cannot process overlapping chromosomes, it can measure touching chromosomes. When the operator recognizes touching chromosomes, a data switch is turned on and 2 points are entered on both sides of the touching area. The computer then automatically separates the chromosomes. This ability to process touching chromosomes is very important since although it is easy to find cells without overlapping chromosomes it is difficult to find cells without touching chromosomes.

Another property of the interactive system is a very small incidence of error of recognition of the centromere. Since the centromere position is displayed on the storage oscilloscope, the operator can always override the computer in a case where an error is suspected. This system is so easy to operate that an operator can be trained in a few hours. 


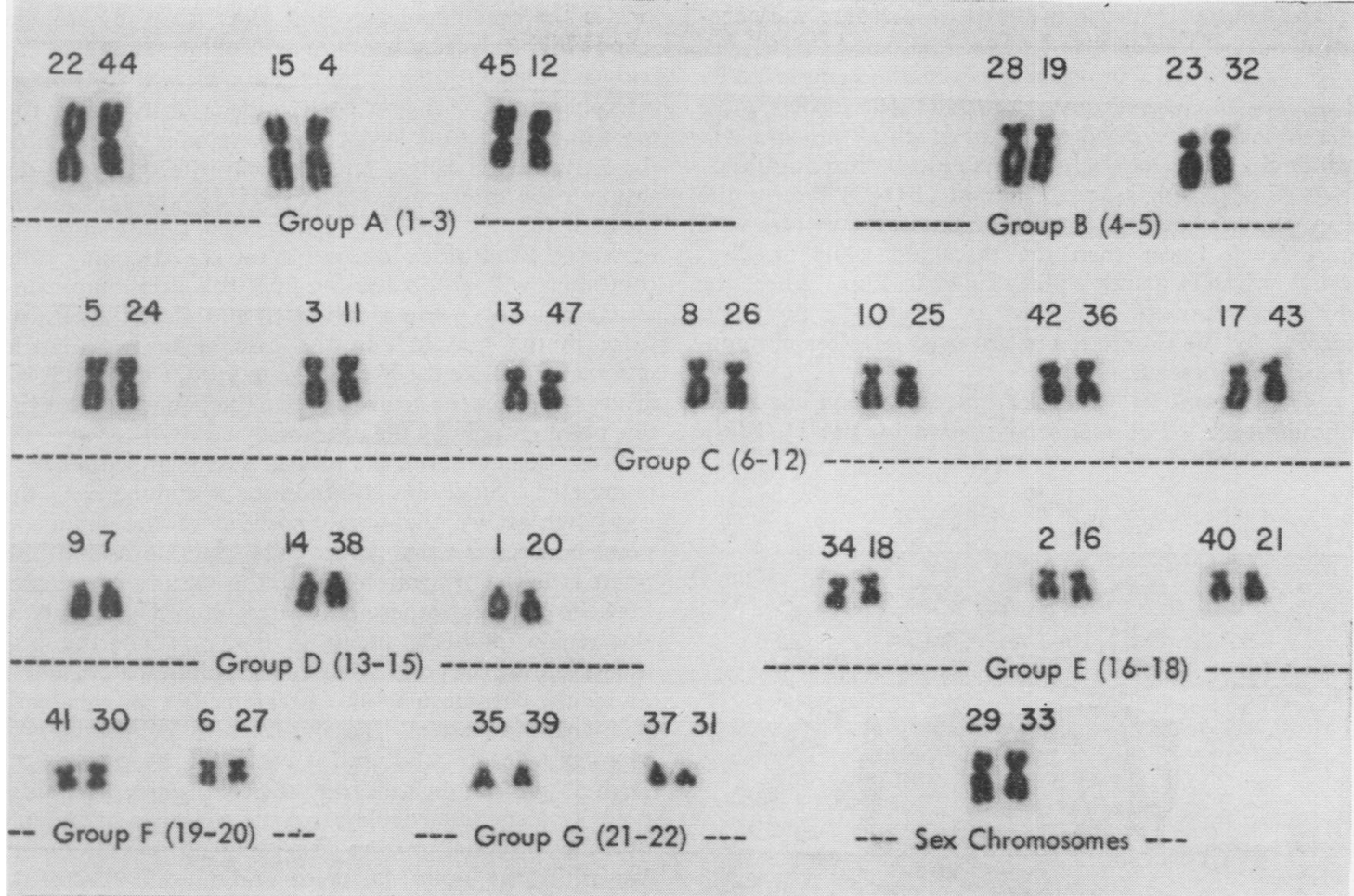

Fig. 3a. A standard female metaphase karyotype. Above each chromosome is the identification number assigned by the computer.

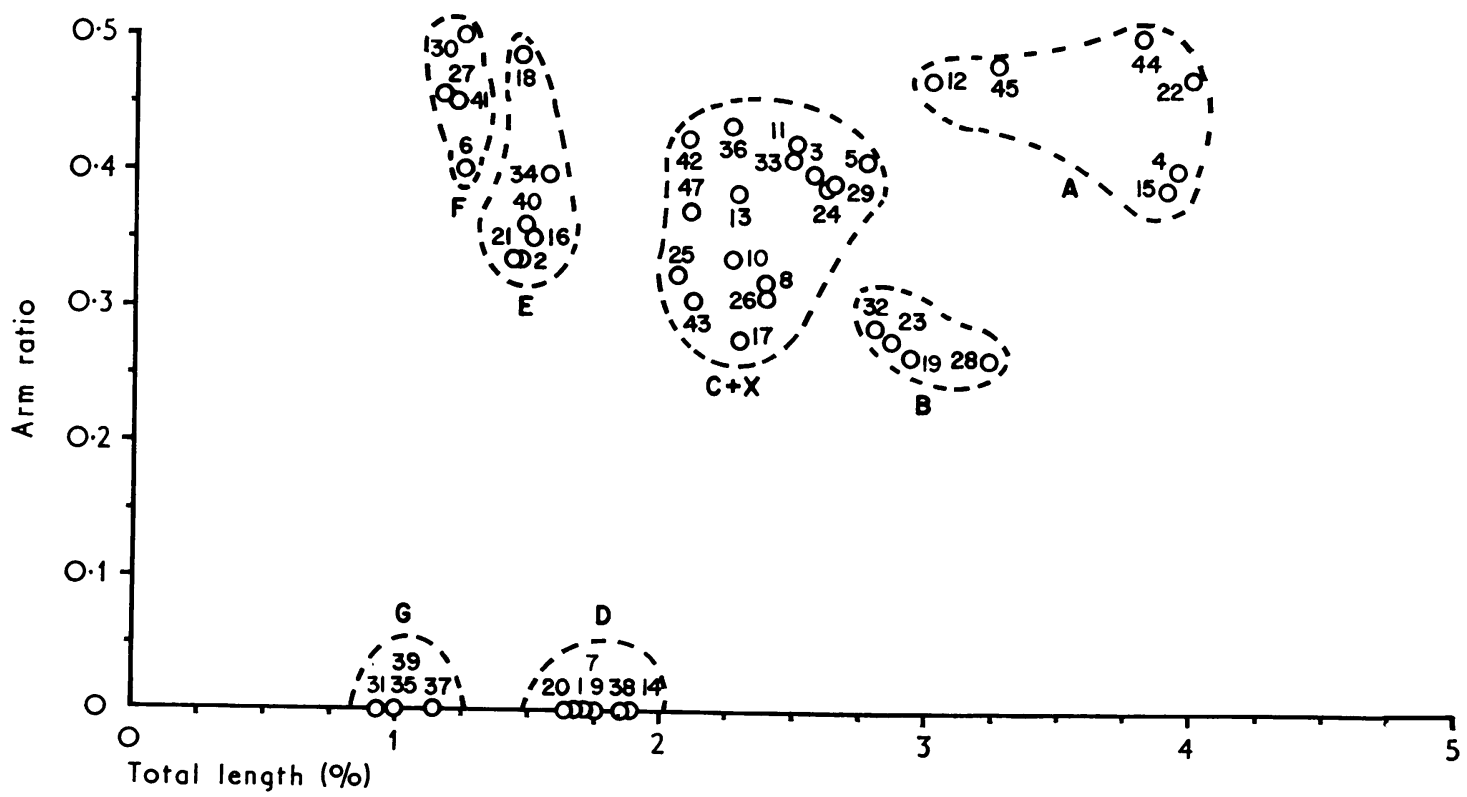

Fig. 3b. Computer plot of chromosome measurements for the normal female in Fig. 3a. The broken line defines major groups according to the Denver classification. The circles represent single chromosomes which have been given identification numbers by the computer.
In some cases the circles overlap. 
Patients. The 2 families described in this paper were initially brought to the attention of the Division of Medical Genetics, Department of Medicine because of an abnormal karyotype (S. P.) and a history of 3 consecutive spontaneous abortions (S. H.).

\section{Results}

Controls. Figure 3a shows the karyotype of a normal female cell performed by an experienced technician and Fig. 3b shows the computer results for the same cell. These analyses were obtained independently since the technician made the karyotype unaware of the computer results. Numbers above each chromosome in Fig. 3a are the ones assigned by the computer (Fig. 1). The technician obtained this numbering from data similar to Fig. 1 and not Fig. 3b. Fig. $3 \mathrm{~b}$ shows clustering of the chromosomes in separately distinctive groups consistent with standard major groups (A, B, C, etc) and a comparison of Fig. 3a with Fig. $3 b$ shows that both methods select the same chromosomes for each of these groups. The pairs A1 $(22,44), A 2$ (4, $15)$, and $A 3(12,45)$ can be clearly identified in Fig. $3 b$ but the $\mathrm{X}$ chromosomes cannot be distinguished since they cluster with the $\mathrm{C}$ group. As mentioned before, the acrocentric chromosomes in groups $\mathrm{D}$ and $\mathrm{G}$ are processed as if they have only 2 arms and therefore the arm ratio is zero. The clustering pattern for length measurements shown in Fig. $3 b$ is consistent with computer results obtained from 24 other cells.

To get an estimate of the precision of our length measurements we have analysed 24 cells using only the unambiguously classified group A chromosomes. The standard deviation for each length measurement is $\sigma_{\mathrm{L}}= \pm 0.08 \%$. We feel that this value is independent of arm length (L) and therefore the estimated error for all measurements; both length (L) and arm ratio (R) can be derived from $\sigma_{\mathrm{L}}$. For example, the estimated error for total length (the sum of the short and long arms) is given by $\Delta_{\mathrm{L}}=\sqrt{ } 2 \sigma_{\mathrm{L}}=0 \cdot 11 \%$.

Patients. The karyotype and computer results for S. P. are shown in Figs. $4 \mathrm{a}$ and $4 \mathrm{~b}$. The karyotype shows what appears to be a translocation in the A group chromosomes, $t(2 q-, 1 q+)$ (Fig. 4a). Further karyotype analysis on all available family members in this kindred revealed a similar translocation in a male sib, the father, and the paternal grandfather, all of whom are clinically normal.

In the computer plot (Fig. 4b), we observed only one $\mathrm{A} 1$ chromosome, No. 45 with $\mathrm{L}=4.3 \pm 0 \cdot 11 \%$ and $\mathrm{R}=0.48 \pm 0.022 \%$ and one $\mathrm{A} 2$ chromosome, No. 36 with $\mathrm{L}=3.7 \pm 0.11 \%$ and $\mathrm{R}=0.40+0.024 \%$ \%
There is one abnormal chromosome, No. 21 with $\mathrm{L}=5.5 \pm 0.11 \%$ and $\mathrm{R}=0.40 \pm 0.016 \%$ and an extra chromosome in the group $\mathrm{C}+\mathrm{X}$ cluster (17 chromosomes instead of 16). This is consistent with a translocation between $\mathrm{A} 1$ and $\mathrm{A} 2$ with the shorter chromosome falling into the $\mathrm{C}+\mathrm{X}$ cluster. The visual karyotype (Fig. 4a) cannot give clear-cut answers to questions regarding translocation of $A 1$ to $A 2$ versus $\mathrm{A} 2$ to $\mathrm{A} 1$ and whether the translocated segment is from the long or short arms. As shown in Fig. 4b, an $\mathrm{A} 1$ to $\mathrm{A} 2$ translocation would involve a translocated length of $5 \cdot 5-3 \cdot 7=2 \cdot 2 \pm 0 \cdot 15 \%$ which would leave the shorter chromosome with $\mathrm{L}=4 \cdot 3-2 \cdot 2=$ $2 \cdot 1 \pm 0 \cdot 2 \%$ and $R=0 \cdot 10 \pm 0 \cdot 10 \%$ (see rectangle I of Fig. 4b). Since there is no extra chromosome in this area, the translocation must therefore be from A2 to A1 involving a translocated length of $1 \cdot 2 \pm$ $0 \cdot 15 \%$. This is equivalent to about one third of the total length of the A2 chromosome.

If this translocation were from the short arms of A2, the shorter chromosome would have $\mathrm{L}=3 \cdot 7-$ $1 \cdot 2=2 \cdot 5 \pm 0 \cdot 2 \%$ and $R=0 \cdot 11 \pm 0 \cdot 11 \%$. Since we do not find a chromosome in this region (see rectangle II, Fig. 4b), the conclusion is that the translocated segment comes from the long arms of A2. In this case, the shorter chromosome would have $\mathrm{L}=2 \cdot 5 \pm$ $0.2 \%$ and $R=0.41 \pm 0.08 \%$ (see rectangle III in Fig. 4b). This region falls within the $C+X$ cluster where we do observe an extra chromosome.

The standard karyotype of the 25-year-old caucasian female $S$. H. is shown in Fig. 5a. This patient has a translocation involving a $\mathrm{D}$ group chromosome. Analysis of other family members revealed the same translocation in the patient's father. All family members are clinically normal and the standard karyotype reveals a $46, \mathrm{XX}, \mathrm{t}(\mathrm{Dq}-$; $\mathrm{Cq}+$ ) pattern. However, the visual analysis could mistakenly interpret the result as a partial deletion of a D group chromosome.

The computer analysis in Fig. 5b shows one more chromosome in both B and $\mathrm{G}$ clusters and one less in clusters $\mathrm{C}+\mathrm{X}$ and $\mathrm{D}$ which makes certain the interpretation of a translocation between a $\mathrm{D}$ group and $\mathrm{C}+\mathrm{X}$ group chromosome. Here the computer results cannot give a clear-cut answer as to whether the translocation is from $C$ to $D$ or $D$ to $C$ since we do not know which one of the $\mathrm{C}$ chromosomes is involved in the translocation.

\section{Discussion}

Reciprocal chromosome translocations are considered in the general category of structural rearrangements that impart little, if any, selective advantage and in many instances no demonstrable abnormalities in the affected individual. Typical 


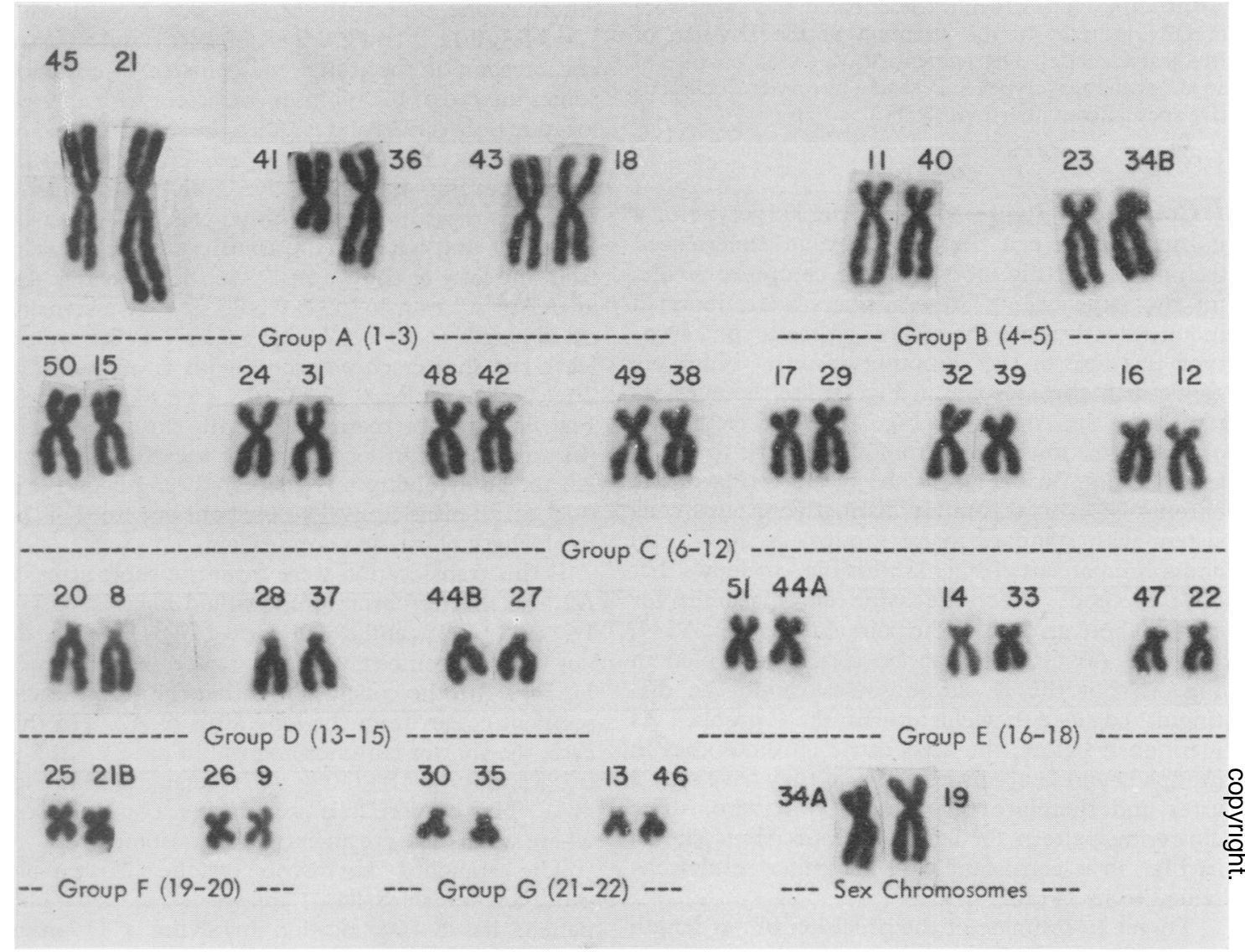

FIG. 4a. A standard metaphase karyotype on patient S. P. as described in Fig. 3a. The translocation involves pairs 1 and 2 in group A.

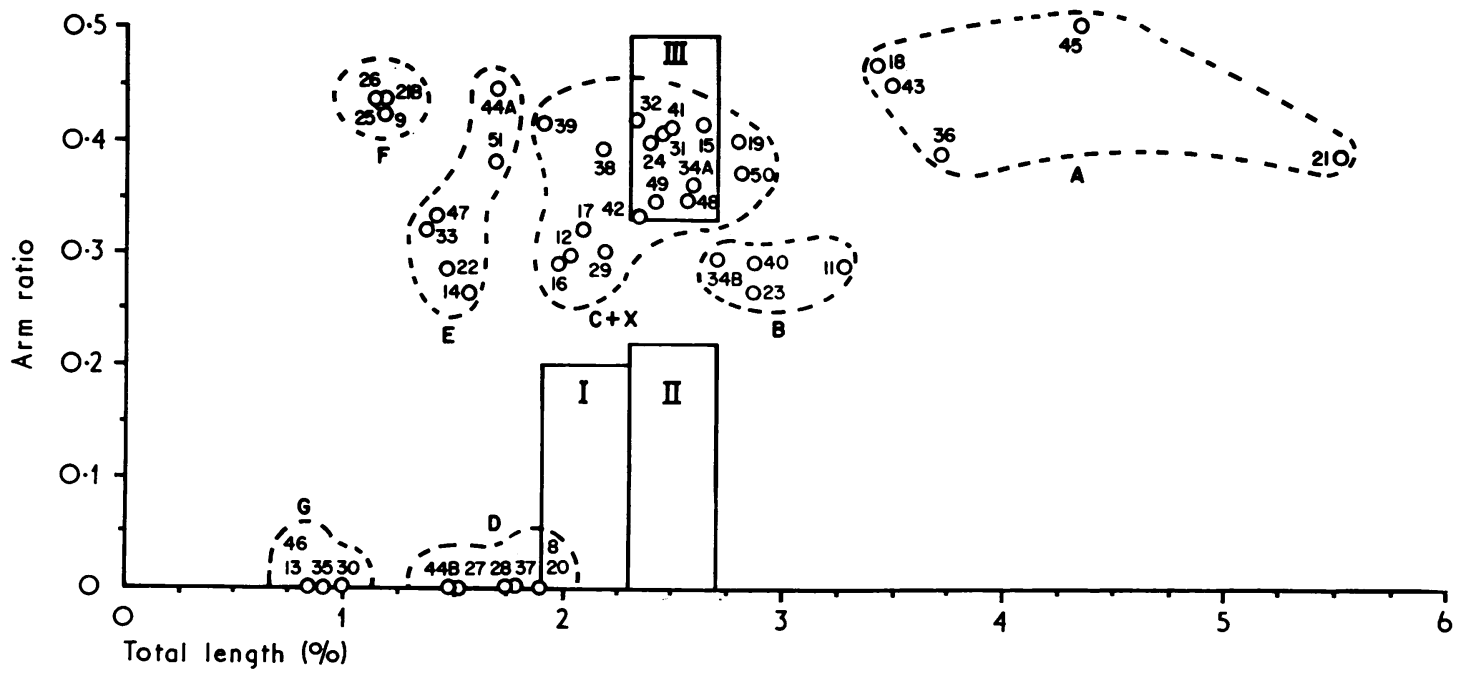

FIG. 4b. Computer plot of chromosome measurements for patient S. P. as described in Fig. 3b. The large solid rectangles I II, and III are described in the results. 


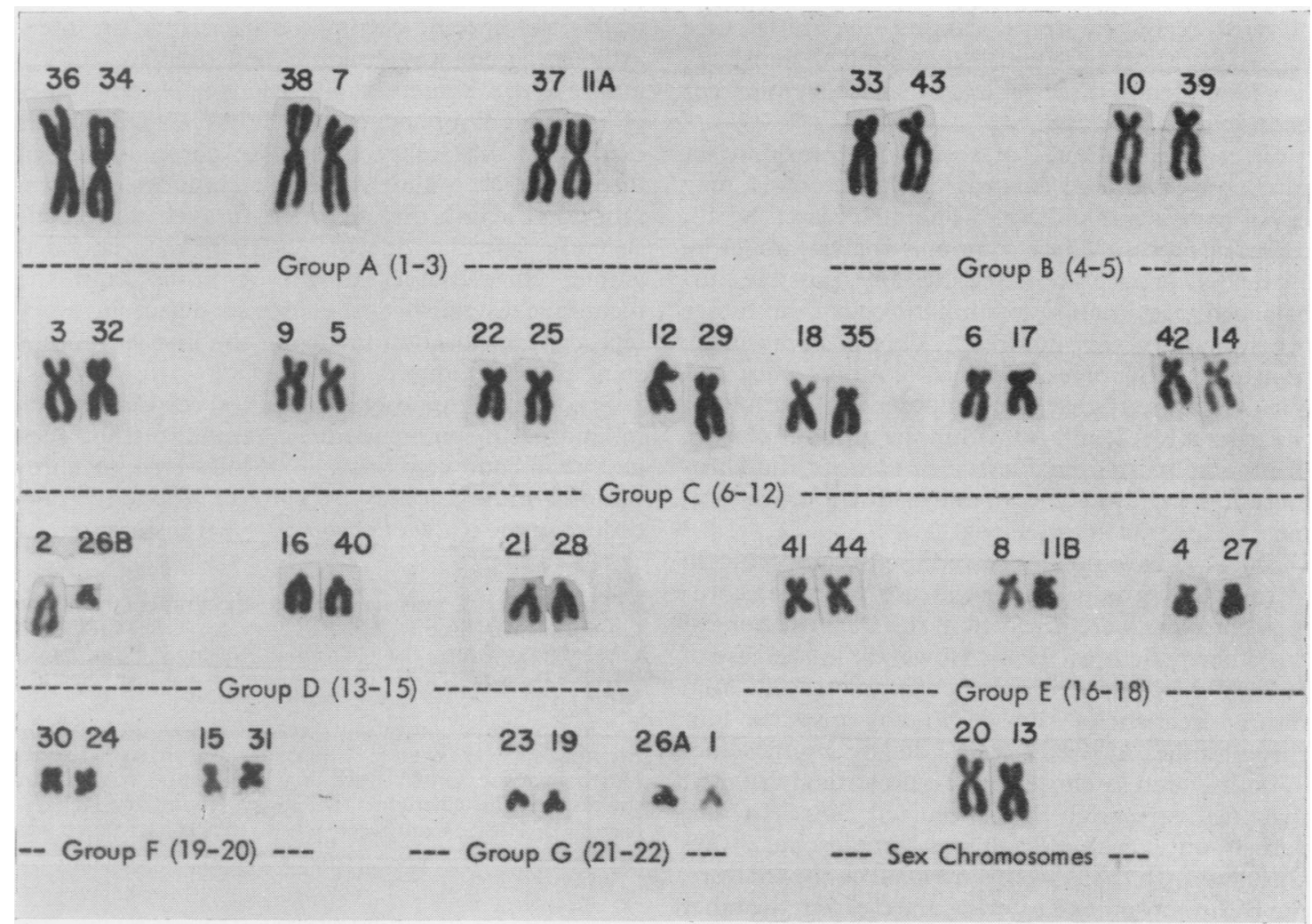

FIg. 5a. A standard metaphase karyotype on patient S. H. as described in Fig. 3a. The translocation probably involves chromosomes $26 \mathrm{~B}$ and 29.

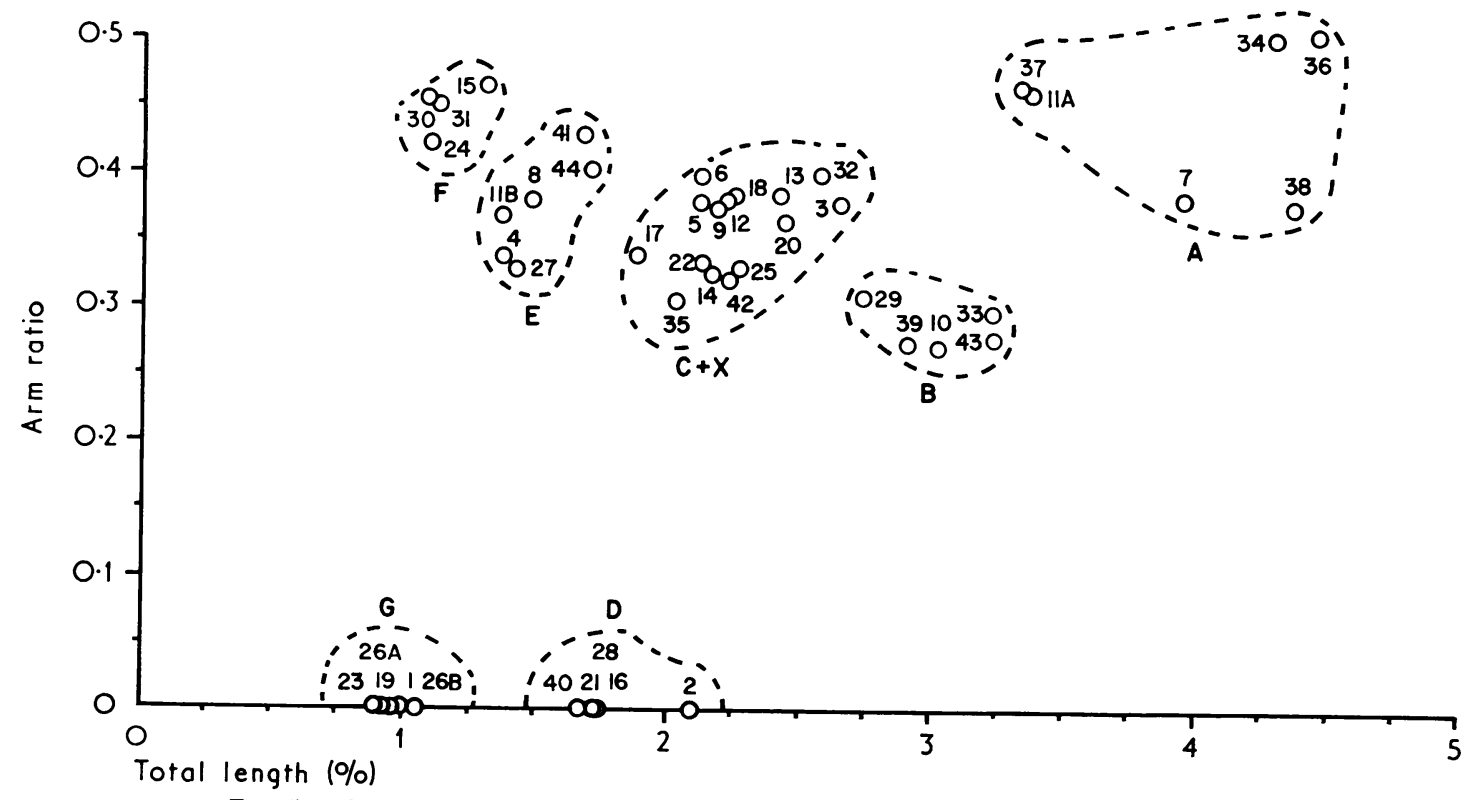

FIG. 5b. Computer plot of chromosome measurements for patient S. H. as described in Fig. 3 b. 
of most reciprocal translocations, the initial case report in this paper describes a familial translocation identified in three generations without apparent phenotypic abnormalities.

In certain examples of reciprocal translocation, computer-orientated analysis of chromosomes may be of considerable help. The first case, S. P., represents a case where computer analysis might be especially precise in detecting any possible unbalanced translocation in amniotic cells from future generations of this kindred. This is because the translocation involves group A chromosomes and our data indicate that the computer can distinguish the pairs A1, A2, and A3 within the group A cluster. Computer pairing enables us to identify the chromosomes involved and obtain a quantitative estimate of the translocated length.

Since we have not yet solved the pairing problem in the other groups, we could not say what chromosome pairs were involved in the translocations of the other patient (S. H.). However, in the case of S. H., if the computer analysis of amniotic cells from future pregnancies showed in any instance four chromosomes in the B cluster and 5 chromosomes in both $\mathrm{D}$ and $\mathrm{G}$ clusters, it would strongly suggest that malsegregation had occurred since in this patient, one structurally altered chromosome always clustered with the B group and in all of our analyses, the B chromosomes cluster separately from the other groups. This defect might not be easily detected by visual karyotype analysis.

The recent reports of more detailed chromosome identification by fluorochrome (Caspersson, Zech, and Johansson, 1970; Caspersson, Lomakka, and Zech, 1971) and modified Giemsa (Drets and Shaw, 1971; Lomholt and Mohr, 1971; Patil, Merrick, and Lubs, 1971) staining raise the possibility of greater sensitivity in computer analysis of chromosome structural rearrangements. The new procedures identify bands of intense staining on long and short arms which appear to be specific for each chromosome pair.

Using fluorochrome staining, Francke (1972) has recently again challenged the dogma that reciprocal translocations involve an exact exchange of chromatin between the 2 chromosomes involved in the translocation.

If this is correct, then even greater precision for chromosome analysis will be required in prenatal fetal karyotyping for families of risk for chromosome malsegregation and it is this area where adjunctive use of computer analysis may be of significant value.

\section{Summary}

To evaluate the usefulness of computer-orientated analysis of specific chromosome variants, an interactive program was designed and used to study 2 kindreds with structural alterations in chromosomes of cultured lymphocytes. One advantage of this method is the ability to analyse chromosomes in direct contact. With available techniques only arm ratio/total length measurements proved to be consistently useful. Specific pairing was obtained among chromosomes of the A group, and the technique established the precise defect in a reciprocal translocation involving the first 2 chromosome pairs of group A.

Studies of chromosome structural rearrangements in kindreds by an interactive computer method suggest that computer analysis of this type of chromosome mutation may be a useful adjunctive procedure in the study of chromosome translocation.

This research was supported by grants from the United States Public Health Service (GM17702 and GM16606), from the National Science Foundation (GB7832), and from the American Cancer Society (E-551).

The authors gratefully acknowledge Mrs Anne Steinbach, Mrs Brenda Sherman, and Miss Virginia Desroches for expert technical assistance. Also our thanks to John Cornelius, Manager, Chemistry Department, IBM 1800 Computer, whose expert assistance was invaluable.

\section{REFERENCES}

Butler, J. W., Butler, M. K., and Stroud, A. (1968). Automatic classification of chromosomes, III. In Data Aquisition and Processing in Biology and Medicine, 5, 21-38, ed. by K. Enslein. Pergamon Press, New York.

Caspersson, T., Lomakka, G., and Zech, L. (1971). The 24 fluorescence patterns of the human metaphase chromosomes-distinguishing characters and variability. Hereditas, Genetiskt Arkiv, 67, 89-102.

Caspersson, T., Zech, L., and Johansson, C. (1970). Differential binding of alkylating fluorochromes in human chromosomes. Experimental Cell Research, 60, 315-319.

Castleman, K. R. (1972). Pictorial output for computerized karyotyping. In Perspectives in Cytogenetics: The Next Decade, ed. by S. W. Wright. Charles Thomas Press, Springfield. (In press.)

Cochrane, I. (1968). Hybrid computer analysis of chromosomal patterns. Biomedical Engineering, 3, 58-65.

Drets, M. E. and Shaw, M. W. (1971). Specific banding patterns of human chromosomes. Proceedings of the National Academy of Sciences, 68, 2073-2077.

Franke, U. (1972). Quinacrine mustard fluorescence of human chromosomes: characterization of unusual translocations. American fournal of Human Genetics, 24, 189-213.

Frey, H. S. (1969). An interactive computer program from chromosome analysis. Computers and Biomedical Research, 2, 274-290.

Gallus, G., Montanaro, N., and Maccacaro, G. A. (1968). A problem of pattern recognition in the automatic analysis of chromosomes: locating the centromere. Computers and Biomedical Research, 2, 187-197.

Gallus, G. and Neurath, P. W. (1970). Improved computer chromosome analysis incorporating preprocessing and boundary analysis. Physics in Medicine and Biology, 15, 435-445.

Hilditch, J. and Rutovitz, D. (1969). Chromosome recognition. Annals of the New York Academy of Sciences, 157, 339-364.

Hungerford, D. A. (1965). Leukocytes cultured from small inocular of whole blood and the preparation of metaphase chromosomes by treatment with hypotonic KCl. Stain Technology, 40, 333-338. 
Ledley, R. S. (1964). High speed automatic analysis of biomedical pictures. Science, 146, 216-223.

Lejeune, J., Dutrillaux, B., and de Grouchy, J. (1970). Reciprocal translocations in human populations: a preliminary analysis. In Human Population Cytogenetics, pp. 81-87, ed. by P. A. Jacobs, W. H. Price, and P. Law. Edinburgh University Press, Edinburgh.

Lomholt, B. and Mohr, J. (1971). Human karyotyping by heatGiemsa staining and comparison with fluorochrome techniques. Nature New Biology, 234, 109-110.

Lubs, H. A. and Ruddle, F. H. (1970). Chromosomal abnormalities in the human population: estimation of rates based on New Haven newborn study. Science, 169, 495-497.

Macintyre, M. N. (1971). Chromosomal problems of intrauterine diagnosis. Birth Defects: Original Article Series, VII, 5, pp. 1826. National Foundation-March of Dimes, New York.

Mendelsohn, M. L., Conway, T. J., Hungerford, D. A., Kolman, W. A., Perry, B. H., and Prewitt, J. M. S. (1966). Computeroriented analysis of human chromosomes. I. Photometric estimation of DNA content. Cytogenetics, 5, 223-242.

Mendelsohn, M. L., Hungerford, D. A., Mayall, B. H., Perry, B. Conway, T., and Prewitt, J. M. S. (1969). Computer-oriented analysis of human chromosomes. II. Integrated optical density as a single parameter for karyotype analysis. Annals of the New York Academy of Sciences, 157, 376-392.

Mendelsohn, M. L., Mayall, B. H., and Prewitt, J. M. S. (1968) Approaches to the automation of chromosome analysis. In Image Processing in Biological Science, pp. 119-136, ed. by D. M. Ramsey. University of California Press, Berkeley.

Neurath, P. W. and Brand, D. H. (1969). Man-machine interaction for image processing. Annals of the New York Academy of Sciences, 157, 324-338.

Patil, S. R., Merrick, S., and Lubs, H. A. (1971). Identification of each human chromosome with a modified Giemsa stain. Science, 173, 821-822.

Ruddle, F., Smith, S., Ledley, R. S., and Belson, M. (1969). Replication-precision study of manual and automatic chromosome analysis. Annals of the New York Academy of Sciences, 157, 400423.

Wald, N. and Preston, K. (1968). Autorratic screening of metaphase spreads for chromosome analysis. In Image Processing in Biological Science, pp. 9-34, ed. by D. M. Ramsey. University of California Press, Berkeley.

Xuong, Nguyen-huu (1969). An automatic scanning densitometer and its application to $x$-ray crystallography. Fournal of Scientific Instruments, 2, Series 2, 485-489. 